\title{
Non-meiotic chromosome instability in human immature oocytes
}

\author{
Gemma Daina ${ }^{1,2}$, Laia Ramos ${ }^{2}$, Mariona Rius ${ }^{2}$, Albert Obradors ${ }^{1,3}$, Javier del Rey ${ }^{1,2}$, Magda Giralt ${ }^{2}$, \\ Mercedes Campillo ${ }^{4}$, Esther Velilla ${ }^{5}$, Aïda Pujol ${ }^{3}$, Olga Martinez-Pasarell ${ }^{6}$, Jordi Benet ${ }^{1,2}$ and \\ Joaquima Navarro ${ }^{\star, 1,2}$
}

\begin{abstract}
Aneuploidy has been a major issue in human gametes and is closely related to fertility problems, as it is known to be present in cleavage stage embryos and gestational losses. Pre-meiotic chromosome abnormalities in women have been previously described. The aim of this study is to assess the whole-chromosome complement in immature oocytes to find those abnormalities caused by mitotic instability. For this purpose, a total of 157 oocytes at the germinal vesicle or metaphase I stage, and discarded from IVF cycles, were analysed by CGH. Fifty-six women, between 18 and 45 years old (mean 32.5 years), including 32 IVF patients (25-45 years of age) and 24 IVF oocyte donors (18-33 years of age), were included in the study. A total of $25 / 157$ (15.9\%) of the oocytes analysed, obtained from three IVF clinics, contained chromosome abnormalities, including both aneuploidy (24/157) and structural aberrations (9/157). Independently of the maternal age, the incidence of abnormal oocytes which originated before meiosis is $15.9 \%$, and these imbalances were found in $33.9 \%$ of the females studied. This work sheds light on the relevance of mitotic instability responsible for the generation of the abnormalities present in human oocytes.
\end{abstract}

European Journal of Human Genetics (2014) 22, 202-207; doi:10.1038/ejhg.2013.106; published online 22 May 2013

Keywords: premeiotic instability; immature oocytes; aneuploidy; segmental imbalances; germline mitotic abnormalities

\section{INTRODUCTION}

Aneuploidy has been a major issue in human gametes, and is highly related to fertility problems. It is known to be present in cleavage stage embryos and gestational losses. ${ }^{1,2}$

The prevailing mechanism causing aneuploidy in humans concerns maternal meiotic mal-segregation. Several studies have focused their interest on oocyte aneuploidy, mainly those which originate during the first and second meiotic divisions. ${ }^{1}$ For this purpose, first and second polar bodies ( $1 \mathrm{~PB}$ and $2 \mathrm{~PB}$ ) and the corresponding metaphase II (MII) oocytes have been analysed, describing aneuploidies of meiotic origin, mainly caused by chromosome non-disjunction and/ or sister chromatid predivision. ${ }^{3}$

Meiotic studies have been performed in oocytes using a great variety of cytogenetic methodologies. R-banding karyotyping on 1397 oocytes showed aneuploidies in $10.8 \%$ of the cells studied. ${ }^{4}$ An aneuploidy rate of $47.5 \%$ was observed when 1PB-MII oocyte doublets were analysed by 9 -chr FISH. ${ }^{5}$ In studies where the wholechromosome complement was assessed by spectral karyotyping, aneuploidy rates from $16.7 \%(10 / 60)^{6}$ to $42.5 \%(20 / 47)^{7}$ were detected. Using comparative genomic hybridisation (CGH), a great variability of aneuploidy rates was found, when different wholegenome amplification methodologies were used to amplify the DNA from single cells, and aneuploidy rates found in 1PB-MII oocyte doublets varied from $3 \%$ in oocyte donors ${ }^{8}$ to $65 \%$ in IVF patients. ${ }^{9}$
Lately, some studies have applied array-CGH and have reported aneuploidy rates of $72 \%{ }^{10}$

About a decade ago, another mechanism causing aneuploidy, apart from meiosis, was seen. This premeiotic mechanism was found to be responsible for the presence of trisomic cells together with the euploid ones, when mature oocytes and embryos from euploid women were analysed. ${ }^{11,12}$ Other authors have confirmed the existence of these premeiotic alterations as non-complementary results between 1PB-MII oocyte doublets in studies where the whole-chromosome complement was evaluated. Non-complementary rates between 1 and $15.5 \%$ have been described. ${ }^{8,13-18}$ It has been postulated that these aneuploid cells could have originated in the early mitotic divisions of the embryo or during mitotic proliferation of the oogonia in embryogenesis. ${ }^{19,20}$ Additionally, direct studies in ovaries of euploid fetuses indicate that ovary mosaicism might be a common situation. ${ }^{20}$

Structural imbalances in oocytes have also been determined by different cytogenetic approaches. Using R-banding on fixed MII oocytes, $2.1 \%$ of the cells showed structural aberrations, including chromatid and chromosome breaks, acentric fragments and deletions. ${ }^{4}$ Recently, using CGH, segmental gains and losses were also identified in $1.9 \%$ of the cells. ${ }^{21}$

To shed some light on oocyte abnormalities which are unexplained by meiotic mechanisms, the aim of this work is to perform a comprehensive aneuploidy screening of immature oocytes either at

${ }^{1}$ Càtedra de Recerca Eugin-UAB, Universitat Autònoma de Barcelona, Bellaterra, Spain; ${ }^{2}$ Unitat de Biologia Cellular i Genètica Mèdica Facultat de Medicina Departament de Biologia Cellular, Fisiologia i Immunologia Universitat Autònoma de Barcelona, Bellaterra, Spain; ${ }^{3}$ Clínica Eugin, Barcelona, Spain; ${ }^{4}$ Laboratori de Medicina Computacional, Unitat de Bioestadistica, Universitat Autònoma de Barcelona, Barcelona, Spain; ${ }^{5}$ nnstitut Marquès Servei de Biologia de la Reproducció, Barcelona, Spain; ${ }^{6}$ Fundació Puigvert Hospital de Sant Pau i de la Santa Creu, Barcelona, Spain

*Correspondence: Dr J Navarro, Unitat de Biologia Cellular i Genètica Mèdica, Departament de Biologia Cellular Fisiologia i Immunologia, Facultat de Medicina, Universitat Autònoma de Barcelona, Bellaterra 08193, Spain. Tel: +34 93581 1724; Fax: +34 93581 1025; E-mail: Joaquima.Navarro@uab.cat

Received 22 October 2012; revised 23 March 2013; accepted 19 April 2013; published online 22 May 2013 
the germinal vesicle or MI stage, in order to determine chromosome abnormalities, either aneuploidies or segmental imbalances, present in the oocytes before entering meiosis.

\section{MATERIALS AND METHODS}

\section{Cell retrieval}

A total of 157 immature oocytes discarded from IVF cycles were analysed by CGH. ${ }^{22}$ The oocytes were donated by 56 women, between 18 and 45 years old. The mean maternal age was 32.5 years of age and included 32 patients (25-45 years of age) and 24 oocyte donors (18-33 years of age). The infertility parameters of the IVF patients included in the study were varied, involving female factors such as advanced maternal age, or gynaecological problems (five cases), male factors such as oligo, astheno or theratozoospermia and/or the combination of them (six cases), male and female factors together (five cases) or other causes (16 cases) including AIH, AID or IVF failure, PGD, serodiscordant couples, idiopathic causes, and so on. The oocyte donors were chosen according to each collaborative IVF centre.

The oocytes were retrieved and donated by three IVF centres in Barcelona, Spain, and each sample was identified with a different code: Clínica Eugin (IME), Institut Marquès (IMM) and Fundació Puigvert (IMP).

Female fibroblasts (46, XX) were cultured to the confluent stage and single cells were used as reference for CGH.

\section{Ethical approval}

According to the legislation in our country, Spain, all IVF patients and donors signed the corresponding informed consent.

\section{Isolation and lysis}

The zona pellucida was removed from each oocyte using Tyrode's acid. Afterwards, the oocytes were washed in three droplets of phosphate bufferedsaline (PBS) with $0.1 \%$ polyvinyl alcohol (PVA). The reference fibroblasts were washed in PBS/0.1\% PVA droplets, isolated individually and placed in PCR tubes for their storage.

The lysis of the cells was performed adding $1 \mu \mathrm{l}$ of SDS $(17 \mu \mathrm{M})$ and $2 \mu \mathrm{l}$ of proteinase $\mathrm{K}(125 \mu \mathrm{g} / \mathrm{ml})$. One droplet of mineral oil was placed in each tube to avoid evaporation. Lysis was performed at $37^{\circ} \mathrm{C}$ for $1 \mathrm{~h}$ followed by a $10 \mathrm{~min}$ step at $95^{\circ} \mathrm{C}$ to inactivate proteinase $\mathrm{K}$.

\section{Comparative genomic hybridisation analysis}

Each cell was amplified, and the DNA was labelled by Nick-Translation; then, CGH was performed as previously described. ${ }^{22}$

The capture of the metaphases was performed by a Nikon eclipse $90 \mathrm{i}$ epifluorescence microscope (Nikon, Melville, NY, USA). A minimum of 12 metaphases were captured per cell and analysed using Isis CGH software developed by MetaSystems (Altlußheim, Germany). Considering that a similar chromosome dosage means euploidy, chromosome losses and gains were considered when the ratio between both labelled DNAs was under or above the limits established by the software according to SD variations. The telomeres and centromeres were excluded from the analysis as they can give misleading profiles. In the cases where chromosomes 17, 19 and 22 altogether were gained or lost in the same cell, they were excluded from the analysis, as they might be artifactual. ${ }^{13,22,23}$

\section{Statistical analysis}

$\chi^{2}$ tests were used to assess whether the differences between the established groups had statistical relevance.

\section{RESULTS}

In this study, 157 immature oocytes were analysed. DNA smears between $200 \mathrm{bp}$ and $4000 \mathrm{bp}$ were observed after whole-genome amplification and electrophoresis for all of the cells, and therefore CGH was performed.

The 157 oocytes analysed by CGH came from 56 different females with an age range between 18 and 45 years of age, the mean being
32.5 years old. Of the 56 females included in this work, 19 produced at least one oocyte with chromosome abnormalities (33.9\%) including aneuploidies and/or segmental imbalances, as detailed in Table 1. Twenty-five oocytes out of 157 (15.9\%) showed chromosome imbalances. (obtained CGH profiles are shown in Supplementary data).

A total of 24 oocytes presented aneuploidies (aneuploidy rate $15.3 \%$ ) for one to eight chromosomes. The total number of aneuploid events in the oocytes analysed was 57 . All chromosomes were involved in aneuploidy except chromosome 14. The highest aneuploidy rate was found for chromosome 21, followed by 13, 16, 19 and 22, as shown in Figure 1.

The use of CGH for comprehensive aneuploidy screening also permitted the detection of structural abnormalities, as shown in Table 1 and Figure 2. Nine out of 157 oocytes showed segmental imbalances $(5.7 \%)$, in eight cases they were accompanied by aneuploidies, and in one oocyte, only a structural imbalance for chromosome 8 was observed.

\section{Age-related aneuploidy}

Considering the oocytes from women aged 36 years of age or less, 102 oocytes from 31 women were analysed. Ten out of the $31(32.3 \%)$ produced at least one cytogenetically abnormal oocyte. Meanwhile, in advanced maternal age (AMA) females ( $\geq 37$ years of age), 55 oocytes from 25 women were evaluated and nine patients (16.4\%) had at least one abnormal oocyte.

The aneuploidy rate in the younger group was $15.7 \%(16 / 102)$, involving 41 whole-chromosome events where the most frequent aneuploidy was for chromosome 16. In the AMA group, the aneuploidy rate was $14.5 \%(8 / 55)$ comprising 16 events, with chromosome 21 being the most frequent.

Segmental chromosome imbalances were found in 5.9\% (6/102) of the oocytes in the younger group, while in the AMA group they were observed in $5.4 \%(3 / 55)$ of the cells.

All data were statistically evaluated by $\chi^{2}$ tests and yielded no significant differences between these groups.

\section{Patient versus donor aneuploidy}

From 32 IVF patients in the study, a total of 83 oocytes could be analysed; at least one abnormal oocyte could be identified in $37.5 \%$ of these patients (12/32). In the oocyte donor group, 74 oocytes from 24 women were assessed, and at least one oocyte was diagnosed as chromosomally abnormal in $29.2 \%$ of the donors (7/24).

In the IVF patient group, $15.7 \%(13 / 83)$ of the oocytes were aneuploid, whereas the donor group had an aneuploidy rate of $14.9 \%$ $(11 / 74)$. The aneuploid events were 35 in patients and 22 in oocyte donors. Chromosome 21 was the one presenting more frequent aneuploidy in patients, while in oocyte donors it was chromosome 16.

Segmental chromosome imbalances were detected in $4.8 \%(4 / 83)$ of the oocytes in the IVF patient group and in $6.7 \%(5 / 74)$ in the oocyte donor group.

The $\chi^{2}$ test was performed to compare the data obtained, and no significant differences were found between these two groups of women.

\section{DISCUSSION}

In the present study, conclusive CGH profiles were obtained for all 157 immature oocytes, achieving a resolution between 10-20 Mb. Not only aneuploidies but also segmental chromosome imbalances between 26.5 and $110 \mathrm{Mb}$ have been detected in $15.9 \%$ of the cells analysed. The incidences observed in the established groups (Table 2) showed no significant differences. 
Table 1 Detailed chromosome abnormalities observed in the oocytes analysed

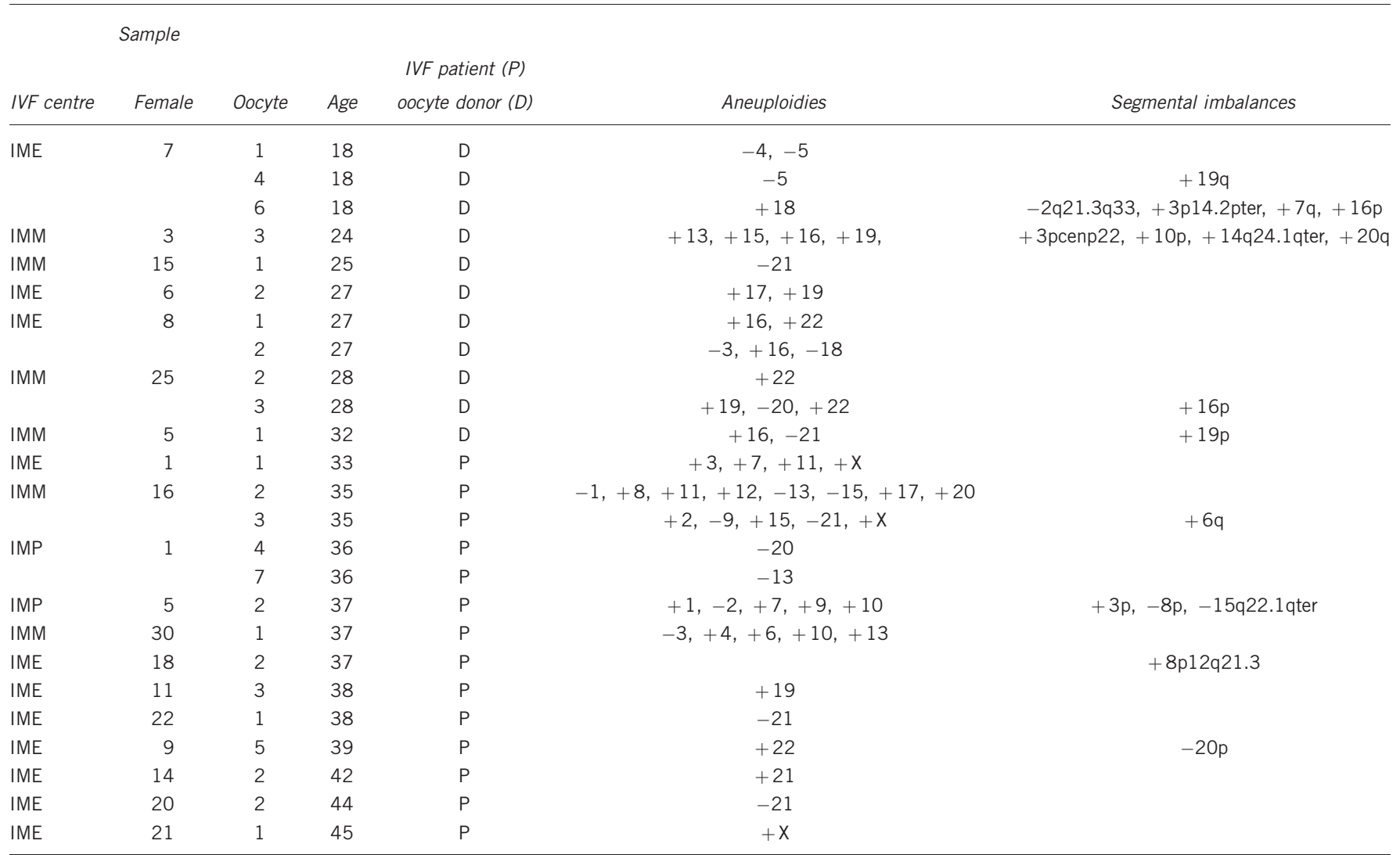

\section{Oocyte aneuploidy}

In terms of aneuploidy, $15.3 \%$ of the oocytes included in this work displayed whole-chromosome imbalances. The mechanisms responsible for the aneuploidy found here cannot be attributed to meiotic origin, such as sister chromatid predivision or bivalent non-disjunction, as all of the oocytes were immature, and meiosis had still not taken place. Therefore, non-meiotic mechanisms must be considered as being responsible for these alterations. The presence of a premeiotic mechanism responsible for aneuploidy was first described after analysing $1 \mathrm{~PB}$ and its corresponding MII oocyte and finding non-complementary results between both cells. ${ }^{12}$ Afterwards, subsequent studies analysing $1 \mathrm{~PB}$ and MII oocyte doublets by $\mathrm{CGH}$ also showed non-complementary results, with heterogeneous incidence, attributable to the existence of precursor aneuploid oogonia not only in IVF patients, but also in oocyte donors, as shown in Table 3. ${ }^{8,13-18}$

The overall aneuploidy rate detected was similar to that previously described in oocyte donors (younger than 29 years of age) and a bit higher than that previously identified in IVF patients (21-42 years of age) (Table 3). The differences observed could be attributable to the size of the samples and the variability among the patients included in each study.

All of the chromosomes, except chromosome 14, were present in the 57 aneuploid events observed. The most frequently involved chromosomes were chromosome 21, followed by 13,16, 19 and 22 . According to these data, it can be inferred that the smallest chromosomes seem to be more commonly affected by premeiotic chromosome segregation errors, similar prevalence of the implication of smaller chromosomes in aneuploidy has also been described as a consequence of errors at the meiotic level. ${ }^{7,14,16,21,24,25}$
In the present work, half of the aneuploid oocytes showed aneuploidies for just one chromosome (Table 1). Contrary to previously published results of analysing oocytes from IVF donors, mitotic errors displayed more than one altered chromosome, while meiotic errors involved single chromosomes only. ${ }^{18}$

\section{Aneuploidy origin}

As stated above, no differences have been observed regarding the aneuploidy rate in immature oocytes depending on maternal age. Accordingly, it can be inferred that the mechanism responsible for this type of events produced during embryonic development would be present in a basal level in all women, having an individual-dependent prevalence rather than being related to maternal age. In fact, a previous work that analysed oocytes from eight euploid fetuses described the presence of aneuploid 21 oogonia, and the authors considered mosaicism in the ovaries as a normal situation in normal female fetuses. ${ }^{20}$

Mitotic chromosome segregation errors found in oocytes could have two different explanations. On the one hand, they could have been produced in the first mitotic divisions of the embryo, where the aneuploid cells were later recruited in certain tissues, in this case the ovaries. On the other hand, they could have originated during the proliferative phase of oogenesis, and maintained in the ovaries due to errors in oogenesis checkpoint mechanisms that should derive them to atresia. ${ }^{26-29}$

Hormonal treatment has also been proposed as a triggering agent in aneuploidy recruitment in different groups of women. A preferential recovery of the aneuploid oocytes with more aneuploid events, which originated from aneuploid oogonia, was found in young females as a possible effect of intensive hormonal stimulation. ${ }^{30-32}$ It has also been postulated that in natural situations, the maturation 

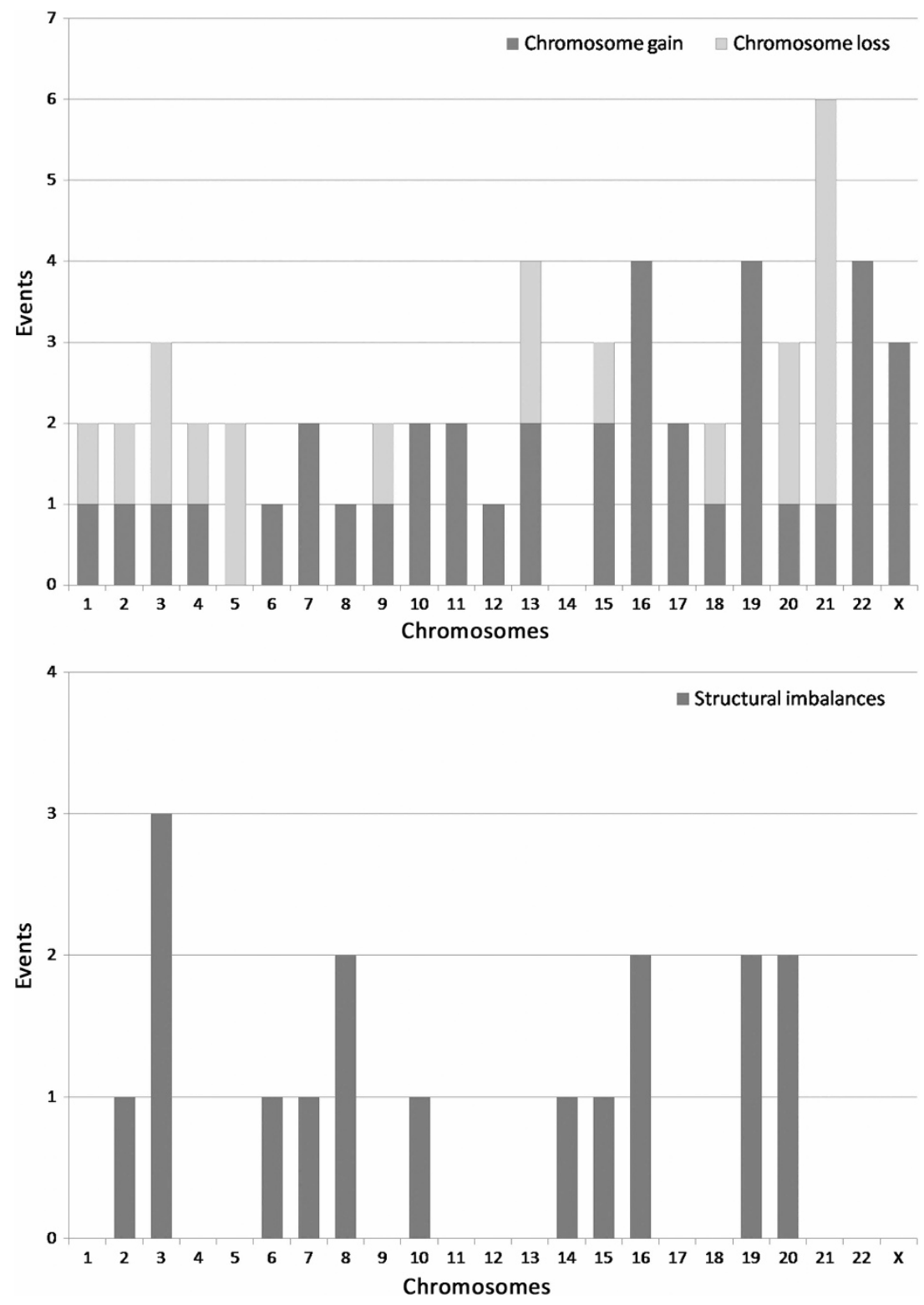

Figure 1 Chromosomes abnormalities and structural imbalances found in the studied oocytes.

of the trisomic oocytes is postponed in relation to the disomic ones. This is the reason why there is an accumulation of these oocytes in the ovarian reserves of older women. ${ }^{20,33}$ To shed light on the effect of hormonal stimulation in relation to natural cycles and, later, conclude whether or not the protocols applied favour aneuploid oocyte recruitment and maturation instead of euploid ones, as has been suggested before, ${ }^{34}$ more research should be performed.

\section{Segmental imbalances}

In this work, $5.7 \%$ of the oocytes showed a total of 17 structural or segmental imbalances involving a great variety of chromosomes (Table 1). To our knowledge, using comprehensive chromosome complement analysis in immature oocytes, this is the first time in which structural chromosome imbalances have been identified. Structural chromosome abnormalities have also been identified using R-banding ${ }^{4}$ and also using $\mathrm{CGH},{ }^{16,21}$ both in mature oocytes. It can be supposed that these imbalances of chromosome fragments can be a consequence of chromosome breakage and loss or duplication of certain chromosome fragments being kept unrepaired. ${ }^{35}$ Accordingly, early in embryogenesis (from week 9 to the 5th month), during the formation of germ cells, a very active cell proliferation takes place, and this could lead to a certain level of genetic instability, reminding what has been observed in some tumours. ${ }^{36}$

All of the ends of the segmental imbalances found in this study, except $-8 \mathrm{p} 12$, are located close to previously described fragile sites of the genome. ${ }^{37}$ Specifically, one of the most active fragile sites, FRA3G, located at 3p14.2, has also been found in this work. As stated before, in situations of DNA replication stress, fragile sites can be problematic for replication, displaying a predisposition to break, and could explain the chromosome instability found in this study. ${ }^{38}$

\section{Incidence of premeiotic errors in women}

Overall, $33.9 \%$ of the women included in this study produced at least one immature oocyte with chromosomal errors that originated before 
meiosis. The mechanism responsible for aneuploidies reported here had been previously described in $25.7 \%$ of the females when analysing MII-1PB doublets from IVM oocytes using 9-chr FISH. ${ }^{5}$ The incidence observed in the present work is higher than the $13.2 \%$ found in IVF patients of 21-42 years of age $\mathrm{e}^{13-15}$ and in $22.6 \%$ of IVF oocytes from donors $<29$ years of age, ${ }^{18}$ where the wholechromosome complement of mature oocytes (1PB-MII doublets) was evaluated.

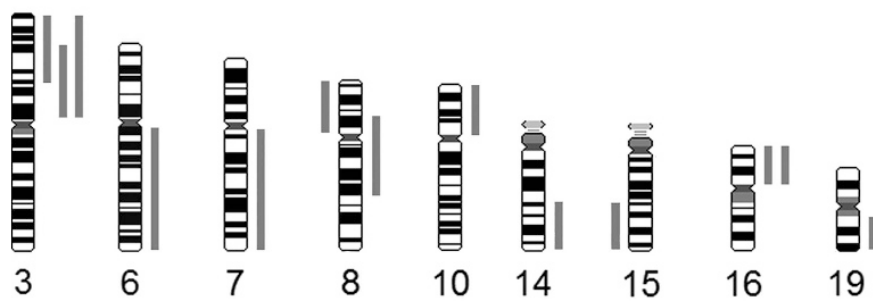

Figure 2 Representation of all the segmental imbalances found in the oocytes. Segmental imbalances on the right of the chromosomes represent gain and to the left represent loss.
The higher incidence detected in the present work could be explained by the fact that all the immature oocytes were evaluated, and it has to be considered that some of the oocytes would have been discarded from previous studies due to their lack of maturation. It is known that some of the aneuploid oocytes would have never gone through the checkpoint at meiosis. ${ }^{39-41}$ Despite the fact that the mitotic origin of abnormal oocytes cannot be used to infer the reproductive risk of women, as not all of them will reach the MII stage, they do allow us to know how relevant this non-meiotic mechanism is for generating abnormal oocytes.

\section{CONCLUSIONS}

This work has analysed the whole-chromosome complement of immature oocytes by CGH and provided a deeper basic knowledge of the aneuploidy present in these cells, identifying oocytes with chromosome abnormalities which had originated before meiosis. These abnormal oocytes with aneuploidies and/or structural imbalances could be related to active cell proliferation stages. It has been demonstrated that one-third of the females included in this study produced at least one of these abnormal oocytes and showed no relation to maternal age or infertility problems, therefore considering this phenomenon more frequent than expected. This study sheds light on the relevance of premeiotic cytogenetic errors in human oocytes.

Table 2 Detailed incidences of abnormal immature oocytes from the groups of the study

\begin{tabular}{|c|c|c|c|c|c|c|c|c|}
\hline & \multicolumn{5}{|c|}{ Women } & \multicolumn{3}{|l|}{ Oocytes with } \\
\hline & $\begin{array}{c}\text { Females } \\
\text { (age range; } \\
\text { mean maternal age) }\end{array}$ & $\begin{array}{c}\text { Total } \\
\text { oocytes }\end{array}$ & $\begin{array}{c}\text { producing } \\
\text { abnormal } \\
\text { oocytes (\%) }\end{array}$ & $\begin{array}{c}\text { Abnormal } \\
\text { oocytes (\%) }\end{array}$ & $\begin{array}{c}\text { Aneuploid } \\
\text { oocytes (\%) }\end{array}$ & $\begin{array}{c}\text { segmental } \\
\text { alterations } \\
\text { (\%) }\end{array}$ & $\begin{array}{l}\text { Whole- } \\
\text { chromosome } \\
\text { events }\end{array}$ & $\begin{array}{c}\text { Segmental } \\
\text { chromosome } \\
\text { events }\end{array}$ \\
\hline \multicolumn{9}{|l|}{ Young females } \\
\hline IVF oocyte donors & $24(18-33 ; 25.2)$ & 74 & $7(29.2)$ & 11 (14.9) & $11(14.9)$ & $5(6.7)$ & 22 & 11 \\
\hline IVF patients & $7(25-36 ; 32.4)$ & 28 & $3(42.9)$ & 5 (17.9) & 5 (17.9) & $1(3.6)$ & 19 & 1 \\
\hline \multicolumn{9}{|l|}{ AMA females } \\
\hline IVF patients & $25(37-45 ; 39.6)$ & 55 & $9(36)$ & $9(16.4)$ & $8(14.5)$ & $3(5.4)$ & 16 & 5 \\
\hline Subtotal young females & $31(18-36 ; 26.84)$ & 102 & $10(32.3)$ & $16(15.7)$ & $16(15.7)$ & $6(5.9)$ & 41 & 12 \\
\hline Subtotal IVF patients & $32(25-45 ; 38)$ & 83 & $12(37.5)$ & 14 (16.9) & $13(15.7)$ & $4(4.8)$ & 35 & 6 \\
\hline Total & $56(18-45 ; 32.5)$ & 157 & 19 (33.9) & 25 (15.9) & $24(15.3)$ & $9(5.7)$ & 57 & 17 \\
\hline
\end{tabular}

Table 3 Data compilation of whole-chromosome analysis by CGH in human oocytes

\begin{tabular}{|c|c|c|c|c|}
\hline Author & Analysed cells & $\begin{array}{l}\text { Total } 1 \text { pb oocytes } \\
\text { doublets }\end{array}$ & $\begin{array}{l}\text { Aneuploid } \\
\text { oocytes (\%) }\end{array}$ & $\begin{array}{c}\text { Germline origin aneuploidy } \\
\text { ocytes/total (\%) }\end{array}$ \\
\hline \multicolumn{5}{|c|}{ Patients (18-45 women age range) } \\
\hline Gutiérrez-Mateo et a/ ${ }^{13}$ & $1 \mathrm{pb}$ oocyte doublets & 25 & $12(48)$ & $2(8)$ \\
\hline Gutiérrez-Mateo et all14 & $1 \mathrm{pb}$ oocyte doublets & 42 & $24(57.1)$ & $4(9.5)$ \\
\hline Gutiérrez-Mateo et a/15 & $1 \mathrm{pb}$ oocyte doublets & 14 & $5(35.7)$ & $1(7.1)^{\mathrm{a}}$ \\
\hline Fragouli et a/17 & $1 \mathrm{pb}$ oocyte doublets & 8 & $4(50)$ & $1(12.5)$ \\
\hline Fragouli et al ${ }^{16}$ & 1 pb oocyte doublets & 100 & $22(22)$ & $1(1)$ \\
\hline Daina et al (present work) & Immature oocytes & 83 & $13(15.7)$ & $13(15.7)$ \\
\hline \multicolumn{5}{|l|}{ Donors $(<33)$} \\
\hline Fragouli et $a^{\beta}$ & $1 \mathrm{pb}$ oocyte doublets & 49 & $2(4.1)$ & 0 \\
\hline Obradors et a/ ${ }^{18}$ & $1 \mathrm{pb}$ oocyte doublets & 84 & $27(32.1)$ & $13(15.5)$ \\
\hline Daina et al (present work) & Immature oocytes & 74 & $11(14.9)$ & $11(14.9)$ \\
\hline
\end{tabular}

also compatible with artifactual loss due to fixation. 


\section{CONFLICT OF INTEREST}

The authors declare no conflict of interest.

\section{ACKNOWLEDGEMENTS}

This study was funded by grants from the Fondo de Investigaciones Sanitarias, Instituto de Salud Carlos III (PI 08/0012, PI 11/00625), Grup de Suport a la Recerca of the Generalitat de Catalunya (2009SGR1107) and Càtedra de Recerca Eugin-UAB. The first author had a pre-doctoral grant from Fundació Crèdit Andorrà (2008-2011).

1 Hassold T, Hunt P: Maternal age and chromosomally abnormal pregnancies: what we know and what we wish we knew. Curr Opin Pediatr 2009; 21: 703-708.

2 Delhanty JD: Inherited aneuploidy: germline mosaicism. Cytogenet Genome Res 2011; 133: 136-140.

3 Angell RR: Predivision in human oocytes at meiosis I: a mechanism for trisomy formation in man. Hum Genet 1991; 86: 383-387.

4 Pellestor F, Andreo B, Arnal F, Humeau C, Demaille J: Mechanisms of non-disjunction in human female meiosis: the co-existence of two modes of malsegregation evidenced by the karyotyping of 1397 in-vitro unfertilized oocytes. Hum Reprod 2002; 17: 2134-2145.

5 Pujol A, Boiso I, Benet J et al: Analysis of nine chromosome probes in first polar bodies and metaphase II oocytes for the detection of aneuploidies. Eur J Hum Genet 2003; 11: 325-336

6 Marquez C, Cohen J, Munne S: Chromosome identification in human oocytes and polar bodies by spectral karyotyping. Cytogenet Cell Genet 1998; 81: 254-258.

7 Sandalinas M, Marquez C, Munne S: Spectral karyotyping of fresh, non-inseminated oocytes. Mol Hum Reprod 2002; 8: 580-585.

8 Fragouli E, Escalona A, Gutierrez-Mateo C et al: Comparative genomic hybridization of oocytes and first polar bodies from young donors. Reprod Biomed Online 2009; 19: 228-237.

9 Sher G, Keskintepe L, Keskintepe M et al: Oocyte karyotyping by comparative genomic hybridization (correction of hybrydization) provides a highly reliable method for selecting 'competent' embryos, markedly improving in vitro fertilization outcome: a multiphase study. Fertil Steril 2007; 87: 1033-1040.

10 Geraedts J, Montag M, Magli MC et al: Polar body array CGH for prediction of the status of the corresponding oocyte. Part I: clinical results. Hum Reprod 2011; 26: 3173-3180.

11 Cozzi J, Conn CM, Harper J et al: A trisomic germ cell line and precocious chromatid segregation leads to recurrent trisomy 21 conception. Hum Genet 1999. 104: 23-28.

12 Mahmood R, Brierley CH, Faed MJ, Mills JA, Delhanty JD: Mechanisms of maternal aneuploidy: FISH analysis of oocytes and polar bodies in patients undergoing assisted conception. Hum Genet 2000; 106: 620-626.

13 Gutierrez-Mateo C, Wells D, Benet J et al: Reliability of comparative genomic hybridization to detect chromosome abnormalities in first polar bodies and metaphase II oocytes. Hum Reprod 2004; 19: 2118-2125.

14 Gutierrez-Mateo C, Benet J, Wells D et al: Aneuploidy study of human oocytes first polar body comparative genomic hybridization and metaphase II fluorescence in situ hybridization analysis. Hum Reprod 2004; 19: 2859-2868.

15 Gutierrez-Mateo C, Benet J, Starke $\mathrm{H}$ et al: Karyotyping of human oocytes by cenM-FISH, a new 24-colour centromere-specific technique. Hum Reprod 2005, 20: 3395-3401.

16 Fragouli E, Wells D, Thornhill A et al: Comparative genomic hybridization analysis of human oocytes and polar bodies. Hum Reprod 2006; 21: 2319-2328.

17 Fragouli E, Wells D, Whalley KM, Mills JA, Faed MJ, Delhanty JD: Increased susceptibility to maternal aneuploidy demonstrated by comparative genomic hybridization analysis of human MII oocytes and first polar bodies. Cytogenet Genome Res 2006; 114: 30-38.
18 Obradors A, Rius M, Cuzzi J et al: Errors at mitotic segregation early in oogenesis and at first meiotic division in oocytes from donor females: comparative genomic hybridization analyses in metaphase II oocytes and their first polar body. Fertil Steril 2010; 93: 675-679.

19 Delhanty JD: Mechanisms of aneuploidy induction in human oogenesis and early embryogenesis. Cytogenet Genome Res 2005; 111: 237-244.

20 Hulten MA, Patel SD, Tankimanova M et al: On the origin of trisomy 21 Down syndrome. Mol Cytogenet 2008; 1: 21

21 Fragouli E, Alfarawati S, Goodall NN, Sanchez-Garcia JF, Colls P, Wells D: The cytogenetics of polar bodies: insights into female meiosis and the diagnosis of aneuploidy. Mol Hum Reprod 2011; 17: 286-295.

22 Rius M, Obradors A, Daina G et al: Reliability of short comparative genomic hybridization in fibroblasts and blastomeres for a comprehensive aneuploidy screening: first clinical application. Hum Reprod 2010; 25: 1824-1835.

23 Voullaire L, Slater H, Williamson R, Wilton L: Chromosome analysis of blastomeres from human embryos by using comparative genomic hybridization. Hum Genet 2000; 106: 210-217.

24 Cupisti S, Conn CM, Fragouli E et al: Sequential FISH analysis of oocytes and polar bodies reveals aneuploidy mechanisms. Prenat Diagn 2003; 23: 663-668.

25 Pellestor F, Andreo B, Arnal F, Humeau C, Demaille J: Maternal aging and chromosomal abnormalities: new data drawn from in vitro unfertilized human oocytes. Hum Genet 2003; 112: 195-203.

26 Tease C, Hartshorne GM, Hulten MA: Patterns of meiotic recombination in human fetal oocytes. Am J Hum Genet 2002; 70: 1469-1479.

27 Nicolaidis P, Petersen MB: Origin and mechanisms of non-disjunction in human autosomal trisomies. Hum Reprod 1998; 13: 313-319.

28 Wolstenholme J, Angell RR: Maternal age and trisomy-a unifying mechanism of formation. Chromosoma 2000; 109: 435-438.

29 Robles P, Roig I, Garcia R et al: Pairing and synapsis in oocytes from female fetuses with euploid and aneuploid chromosome complements. Reproduction 2007; 133: 899-907.

30 Weghofer A, Munne S, Brannath W et al: The impact of LH-containing gonadotropins on diploidy rates in preimplantation embryos: long protocol stimulation. Hum Reprod 2008; 23: 499-503.

31 Weghofer A, Munne S, Brannath W et al: The impact of LH-containing gonadotropin stimulation on euploidy rates in preimplantation embryos: antagonist cycles. Fertil Steril 2009; 92: 937-942.

32 Obradors A, Rius M, Daina G, Ramos L, Benet J, Navarro J: Whole-chromosome aneuploidy analysis in human oocytes: focus on comparative genomic hybridization. Cytogenet Genome Res 2011; 133: 119-126.

33 Hulten MA, Patel S, Jonasson J, Iwarsson E: On the origin of the maternal age effect in trisomy 21 Down syndrome: the Oocyte Mosaicism Selection model. Reproduction 2010; 139: 1-9.

34 Handyside AH, Montag M, Magli MC et al: Multiple meiotic errors caused by predivision of chromatids in women of advanced maternal age undergoing in vitro fertilisation. Eur J Hum Genet 2012; 20: 742-747.

35 Voet T, Vanneste E, Van der Aa N et al: Breakage-fusion-bridge cycles leading to inv dup del occur in human cleavage stage embryos. Hum Mutat 2011; 32: 783-793.

36 Voet T, Vanneste E, Vermeesch JR: The human cleavage stage embryo is a cradle of chromosomal rearrangements. Cytogenet Genome Res 2011; 133: 160-168.

37 Mrasek K, Schoder C, Teichmann AC et al: Global screening and extended nomenclature for 230 aphidicolin-inducible fragile sites, including 61 yet unreported ones. Int J Oncol 2010; 36: 929-940.

38 Franchitto A, Pichierri P: Understanding the molecular basis of common fragile sites instability: role of the proteins involved in the recovery of stalled replication forks. Cell Cycle 2011; 10: 4039-4046.

39 Hassold T, Hunt P: To err (meiotically) is human: the genesis of human aneuploidy. Nat Rev Genet 2001; 2: 280-291.

40 Vogt E, Kirsch-Volders M, Parry J, Eichenlaub-Ritter U: Spindle formation, chromosome segregation and the spindle checkpoint in mammalian oocytes and susceptibility to meiotic error. Mutat Res 2008; 651: 14-29.

41 Wang WH, Sun QY: Meiotic spindle, spindle checkpoint and embryonic aneuploidy. Front Biosci 2006; 11: 620-636.

Supplementary Information accompanies this paper on European Journal of Human Genetics website (http://www.nature.com/ejhg) 